\title{
Focus on Proteomics in Honor of the 2002 Distinguished Contribution in Mass Spectrometry Award to W. J. Henzel, J. T. Stults, and C. Watanabe
}

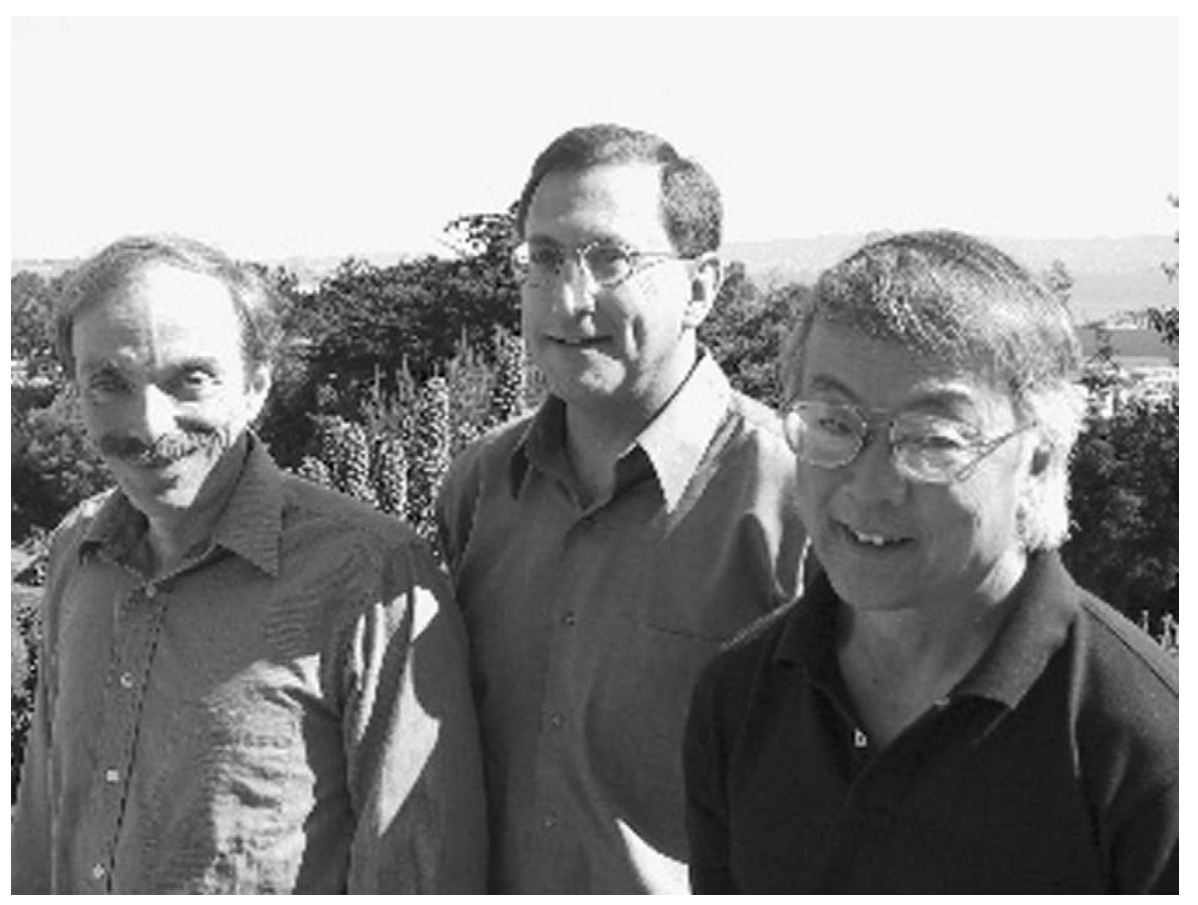

Most of the articles published in this journal, or in any journal, add in an incremental way to the scientific edifice we are committed to create. Sometimes, however, a single article has major impact; it sets a new direction, a new way of thinking, a new design for the future. The article, "Identifying Proteins from TwoDimensional Gels by Molecular Mass Searching of Peptide Fragments in Protein Sequence Databases" by W. J. Henzel, T. M. Billeci, J. T. Stults, S.C. Wong, C. Grimley, and C. Watanabe published in 1993 in the Proc. Natl. Acad. Sci. USA is one such article. The reason for the impact is best expressed in the abstract of the article. The authors are reporting on the opportunity to identify proteins by measuring the masses of a few of the peptides that are generated by digestion of the protein. This is the analytical foundation of Proteomics.

"A rapid method for the identification of known proteins separated by two-dimensional gel electrophoresis

Published online July 31, 2003

\begin{abstract}
is described in which molecular masses of peptide fragments are used to search a protein sequence database. The peptides are generated by in situ reduction, alkylation, and tryptic digestion of proteins electroblotted from two-dimensional gels. Masses are determined at the subpicomole level by matrix-assisted laser desorption/ionization mass spectrometry of the unfractionated digest. A computer program has been developed that searches the protein sequence database for multiple peptides of individual proteins that match the measured masses... This method facilitates simultaneous processing of a large number of two-dimensional gel spots. The method was applied to a twodimensional gel of a crude Escherichia coli extract that was electroblotted onto poly(vinylidene difluoride) membrane. Ten randomly chosen spots were analyzed. With as few as three peptide masses, each protein was uniquely identified from over 91,000 protein sequences..."
\end{abstract}

The article has had impact because it describes for us the role of mass spectrometry in proteomics. Evidence of this impact is found by an inquiry to the ISI Web of Knowledge; the article has close to 500 citations. 
So it is more than appropriate that Henzel, Stults, and Watanabe, the three senior authors of the article, should win the 2002 American Society for Mass Spectrometry Award for Distinquished Contribution. We at the Journal of the American Society of Mass Spectrometry send our congratulations to the awardees by sponsoring a focus on proteomics, a focus that is headlined by an Account and Perspective by Henzel, Watanabe, and Stults in which they describe the genesis of the idea, "peptide mass fingerprinting" starting in 1989 and show how MALDI made its implementation possible.

The Account is followed by nine articles. In the first, Peter Jungblut and coworkers show how "peptide mass fingerprinting" can be improved by elimination of contaminant and other unwanted peptide peaks in the mass spectrum, resulting in considerable improvement in the fingerprinting. John Yates and coworkers compare, in the second article in the focus, the ability of MALDI-TOF and capillary LC hyphenated with tandem mass spectrometry to examine over one hundred protein spots that were separated by 2-D gel electrophoresis. A key step is the clean-up of the protein digest before MS analysis. The two mass spectrometric methods are comparable although the capillary HPLC/ MS/MS method gives better protein sequence coverage than does MALDI TOF. In a sequel and similar article, W. M. Bodnar, M. A. Moseley, and coworkers find that there is significant overlap between the ESI and the MALDI approaches-more than one half of the proteins identified by one method are found by the other. Both methods also uncover unique peptides.

Another important idea in proteomics is demonstrated by Richard Smith and coworkers in an article in which they report the effectiveness of a combination of accurate mass of peptides in a digest and accurate elution times in capillary liquid chromatography. The combination yields, in the vast majority of cases, unique matches for the original protein from a microbial source. Continuing the theme of important improvements in mass spectrometry methodology in proteomics, Laugesen and
Roepstorff show the combinations of matrices give improved sequence coverage and better spot-to-spot reproducibility in MALDI of peptide digests.

Sickmann and coworkers show in the sixth article of the focus the utility of multidimensional capillary HPLC for analysis of protein complexes. This article meets the challenge faced by all who work with protein mixtures; that is, the mixtures of peptides that result from a digest can be exceedingly complex. Sometimes, owing to inadequacies of databases, the peptides produced in a digest must be sequenced de novo. This, of course, requires product-ion spectra that are complete. Chait and coworkers demonstrate that de novo sequencing is possible for peptide sequences of up to 14 amino-acid residues in length by using MALDI ion-trap technology.

The eighth and ninth articles deal with the problems of post-translational modifications. In the first of this pair, Richard Johnson and colleagues at Amgen report an application of HPLC and tandem mass spectrometry to identify tryptic phosphopeptides and their sites of phosphorylation in epidermal growth factor. In the second, Jack Throck Watson and coworkers report an efficient method for data processing and interpretation, an approach they call "negative signature mass," to rule out various theoretical disulfide linkages in peptides containing up to four cystines.

We believe that this collection of articles is a fitting honor to the Henzel, Stults, and Watanabe trio who recognized the role of mass spectrometry in identifying proteins. The research continues in a profound way with the development that they imagined nearly a decade ago. We thank the authors for their participation and send to the three awardees our heartiest and grateful congratulations.

Carol Robinson Associate Editor

Michael Gross Editor 